\title{
QUANTUM-CIRCUIT ANALOG OF THE DYNAMICAL CASIMIR EFFECT AT FINITE TEMPERATURES
}

\author{
SHIGEMASA MATSUO ${ }^{a, b}$ and TOSHIYUKI FUJII ${ }^{a, b} *$, and NORIYUKI HATAKENAKA ${ }^{a}$ \\ ${ }^{a}$ Graduate School of Integrated Arts and Sciences, Hiroshima University, \\ HIgashi-hiroshima, 739-8521, Japan \\ ${ }^{b}$ Research Institute for Science and Engineering, Waseda University, Shinjuku-ku, Tokyo, \\ 169-8555, Japan
}

Published 28 July 2012

\begin{abstract}
We proposed a quantum-circuit analog of dynamical Casimir effect by using a superconducting quantum circuit. The proposed system can be regarded as a time-dependent harmonic oscillator, resulting in squeezing of a quantum flux trapped in the system. Here we develop our theory taking into account finite temperatures, in order to contact with experiments like circuit-QED. We present numerical results of occupation probability distributions produced by non-stationary boundary effect at various temperatures.
\end{abstract}

Keywords: dynamical Casimir effect; circuit QED; Josephson artificial atom.

\section{Introduction}

Absence of matter in space is termed as vacuum in classical mechanics. Quantum mechanics modifies this definition as the state with the lowest energy and predicts that vacuum energy is different from classical value due to the quantum correction to the energy leading to vacuum fluctuation by virtual particles as a brief existence. Vacuum fluctuations may also be related to the so-called cosmological constant in cosmology. The best evidence for vacuum fluctuations of the electromagnetic fields is the Casimir effect that exhibits an attractive force between two perfectly conducting parallel plates when placed in the vacuum. ${ }^{1}$ This was confirmed experimentally by Lamoreaux ${ }^{2}$ and by Mohideen et al. ${ }^{3}$

The remarkable nature of vacuum fluctuation has led to the idea of a dynamical counterpart of the Casimir effect. It predicts photon creation out of vacuum as a result of a non-adiabatic effect caused by highly moving boundaries such as walls

*Present address, Department of Physics, Asahikawa Medical University, Midorigaoka-higashi, Asahikawa, 078-8510, Japan. 
of the electromagnetic cavity. ${ }^{4}$ Despite the significant effort, no experimental verification of dynamical Casimir effect has ever been achieved in this simple scenario, mainly due to the difficulty in moving the mirror at a fast enough speed. ${ }^{5}$

On one hand, there is another procedure that can rapidly change the field boundary conditions effectively that is, the use of either lasers to modulate the reflectivity of a thin semiconductor film ${ }^{6,7}$ or the resonance frequency of a superconducting strip line resonator. ${ }^{8}$ The analogue of the dynamical Casimir effect has been proposed by Dodonov et. al. using superconducting devices. ${ }^{9}$ Along this direction, we proposed quantum-circuit analogue of the dynamical Casimir effect based on a superconducting quantum interference device (SQUID), which is regarded as a superconducting artificial atom. ${ }^{10} \mathrm{~A}$ similar system with different boundary condition has also been proposed. ${ }^{11}$ We have presented both numerically ${ }^{12}$ and theoretically ${ }^{13,14}$ that the quantum states of the quantum flux induced by the non-stationary boundary effect are squeezed states.

In this paper, we investigate the effect of finite temperatures on the nonstationary boundary effect in double SQUID and thereby present specific features in occupation probability distributions induced by non-stationary boundary effect at various temperatures.

\section{Non-adiabatic quantum-state evolutions at finite temperatures}

The system we consider is a double rf-SQUID shown in Fig. 1 (a), which consists of a superconducting loop interrupted by a dc-SQUID. This device behaves as a normal rf-SQUID with tunable Josephson critical current controlled by an external magnetic flux $\Phi_{c}$ threading in a small loop or equivalently called the tunable Josephson plasma frequency $\omega_{J}$. Figure 1 (b) shows the potential profile as a function of $\Phi_{c}$ with different control flux values. This clearly shows the $\Phi_{c}$-dependent Josephson plasma frequency that is roughly proportional to the curvature at the bottom of the potential. Under the time-dependent $\phi_{c}$ situations, the Josephson plasma frequency becomes time-dependent. Thus, the system can be regarded as a time-dependent anharmonic oscillator. The system is further approximated by a time-dependent harmonic oscillator when the charging energy of the junction $E_{c}$ is less than the Josephson coupling energy $E_{J}\left(E_{c}<E_{J}\right)$.

The time-dependent nature of the harmonic oscillator induces a nonlinear term indicating nonadiabatic effect as a result of the Bogoliubov transformation between eigenstates at different times, namely, $b(t)=\hat{V}(t) \hat{a} \hat{V}(t)^{\dagger}$ with $V(t)=$ $\exp \left[-\mu(t)\left(\hat{a}^{2}-\hat{a}^{\dagger 2}\right) / 2\right]$ and $\mu(t)=-\ln (\omega(t) / \omega(0)) / 2$. This term is a source for generating the vacuum squeezed state. In fact, we derived an analytical formula ${ }^{13,14}$ for quantum-state evolution $|\phi\rangle$ under the time-dependent harmonic potential;

$$
|\phi(t)\rangle=e^{-\frac{i}{2} \int_{0}^{t} \omega\left(t^{\prime}\right) d t^{\prime}} e^{-\frac{1}{2}\left\{\nu \hat{b}(t)^{2}-\nu^{*} \hat{b}^{\dagger}(t)^{2}\right\}}|0(t)\rangle
$$


(a)

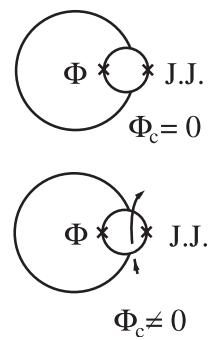

(b)

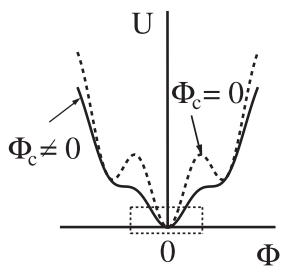

(c)

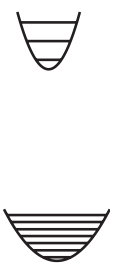

Fig. 1. Schematics and potential energy of Josephson artificial atoms (a) Schematics of a double SQUID with (lower) and without (upper) a magnetic flux in a small loop, (b) Potential energy of the double SQUID at the corresponding $\Phi_{c}$ values, (c) Magnified view at the bottom of the potential energy curve with discrete energy level

with the squeezing parameter

$$
\nu(t)=\frac{1}{2} \int_{0}^{t} \frac{\dot{\omega}\left(t^{\prime}\right)}{\omega\left(t^{\prime}\right)} e^{2 i \int_{t}^{t^{\prime}} \omega\left(t^{\prime \prime}\right) d t^{\prime \prime}} d t^{\prime} .
$$

Note that the squeezing parameter contains the nonadiabatic factor $\dot{\omega}\left(t^{\prime}\right) / \omega\left(t^{\prime}\right)$. This clearly shows the squeezing is connected to the non-adiabatic effect as a result of the rapid boundary changes. Therefore, detecting the squeezing nature is a strong evidence for confirming the dynamical Casimir effect.

In the previous paper, we have investigated quantum-circuit analog of dynamical Casimir effect at an absolute temperature. Our study here in mainly focused at finite temperatures to relate with real experimental situations. In principle, the change of the system's potential distorts thermal distribution of the environment as a result of their inevitable interaction, which further leads to the redistribution of their occupation numbers to attain a thermal equilibrium. However, let us suppose here that the potential change is too fast to modify the thermal distribution. In other words, we assume that the environment with a large number of degrees of freedom encircled the system is frozen under the rapid changes of the potential.

The occupation number probability at the $n$-th quantum state $P_{n}(t)$ is given as $P_{n}(t)=\langle n(t)|\rho(t)| n(t)\rangle$ where $\rho(t)$ is a density matrix expressed as $\rho(t)=$ $\hat{V} \hat{U}_{0} \hat{U}_{\text {int }} \rho(0) \hat{U}_{i n t}^{\dagger} \hat{U}_{0}^{\dagger} \hat{V}^{\dagger}$ with being $U_{0}$ for an unitary operator transferring interaction picture and $U_{\text {int }}$ for a time-translational operator based on the interaction Hamiltonian, i.e., $\hat{H}_{i n t}=i \hbar \dot{\mu}\left(\hat{a}^{2}-\hat{a}^{\dagger 2}\right) / 2$, where $\hat{a}\left(\hat{a}^{\dagger}\right)$ is an annihilation (creation) 
operator and $\dot{\mu}(t)=-(1 / 2) d\{\ln (\omega(t) / \omega(0)\} / d t=-\dot{\omega}(t) / 2 \omega(t)$. Then,

$$
\begin{aligned}
P_{n}(t) & =\left\langle n(t)\left|\hat{V} \hat{U}_{0} \hat{U}_{i n t} \hat{V}^{\dagger}\left\{\hat{V} \rho(0) \hat{V}^{\dagger}\right\} \hat{V} \hat{U}_{i n t}^{\dagger} \hat{U}_{0}^{\dagger} \hat{V}^{\dagger}\right| n(t)\right\rangle \\
& =\left\langle n(t)\left|\hat{V} \hat{U}_{0} \hat{U}_{i n t} \hat{V}^{\dagger} e^{-\frac{\hbar \omega_{0}}{k_{B} T}\left(\hat{b}^{\dagger} \hat{b}+\frac{1}{2}\right)}\left\{\sum_{m}|m(t)\rangle\langle m(t)|\right\} \hat{V} \hat{U}_{i n t}^{\dagger} \hat{U}_{0}^{\dagger} \hat{V}^{\dagger}\right| n(t)\right\rangle \\
& =\frac{1}{Z} \sum_{m}|\langle n(t)|S(\nu(t))| m(t)\rangle|^{2} e^{-\frac{\hbar \omega_{0}}{k_{B} T}\left(m+\frac{1}{2}\right)}
\end{aligned}
$$

where $S(\nu)=\exp \left(\nu \hat{b}^{\dagger 2}-\nu^{*} \hat{b}^{2}\right)$ and $Z$ is the partition function. We also use the relation $\hat{V} f\left(\hat{a}, \hat{a}^{\dagger}\right) \hat{V}^{\dagger}=f\left(\hat{b}, \hat{b}^{\dagger}\right)$. The transition matrix element $\langle n(t)|S(\nu(t))| m(t)\rangle$ is given by Venkata. ${ }^{15}$

\section{Single-step frequency modulation}

Let us investigate the quantum-state evolutions for a flux particle in a double SQUID for a single-step frequency modulation given as $\omega\left(t-t_{0}\right)=\left(\omega_{0}+\omega_{1}\right) / 2-\left(\omega_{0}-\right.$ $\left.\omega_{1}\right) / 2 \tanh \left\{2\left(t-t_{0}\right) / \tau\right\}$ where $\omega_{0(1)}$ is an initial (a final) frequency. $\tau$ and $t_{0}$ are a duration time and an initial time for the frequency modulation, respectively.

Figure 2 shows the occupation probability distribution after the frequency change. Firstly, analytical solutions (triangles) are well consistent with numerical results (bars). Thus, the analytical treatment in the previous section is verified and supported with this consistency. Secondly, a rare even-occupation distribution with Hermite amplitude peculiar to vacuum squeezed state appears at $T=0$ (see Fig. $2(\mathrm{a})$ ) and diminishes at high temperature (see Fig. 2 (c)) . There are no significant changes from the initial Boltzmann distribution. However, we may obtain the squeezing features by investigating the tiny differences. One can find more interesting feature in the intermediate temperatures (see Fig. 2 (b)), namely, the occupation probability at 2 nd quantum state is greater than that at 1 st quantum state. The

(a)

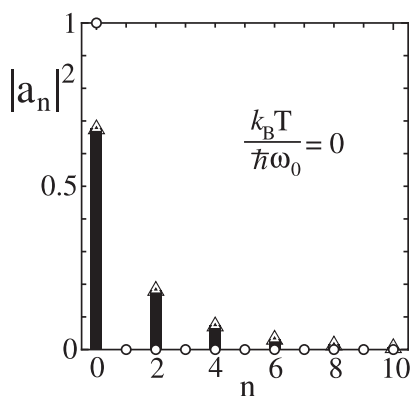

(b)

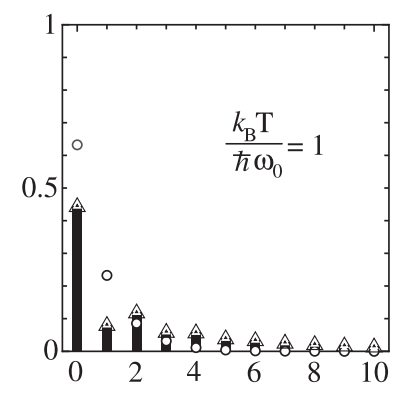

(c)

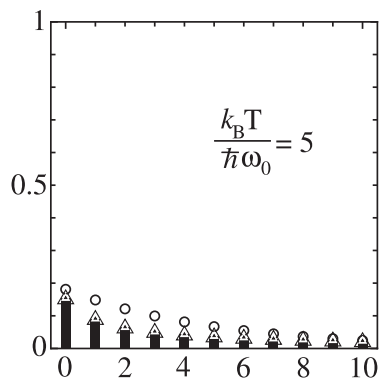

Fig. 2. The occupation probability distribution after the change of Josephson plasma frequency at various temperatures; (a) $k_{B} T / \hbar \omega_{0}=0$ (b) $k_{B} T / \hbar \omega_{0}=1$ and (c) $k_{B} T / \hbar \omega_{0}=5$. The triangles and bars denote analytical and numerical results, respectively. The circles indicate the initial occupation probability to see the differences easier. 
unique nature (the population inversion) is not only useful for confirming squeezed features in this system but can also extend to other application such as a laser action.

\section{Summary}

We have investigated a quantum-circuit analog of dynamical Casimir effect by using a superconducting quantum circuit at finite temperatures treated in both analytic and numerical techniques. An analytical formula has been derived for occupation number distributions due to quantum-circuit analog of dynamical Casimir effect, which has also described well the numerical results. In conclusion, the rare occurrence of occupation probability distribution to squeezing demonstrates a significant signature for dynamical Casimir effect even at finite temperatures.

\section{Acknowledgments}

We are grateful to B. B. Faustino, S. Kurihara and A. Zeilinger for valuable discussions. This work was supported in part by a grant from the Research Foundation for Opto-Science and Technology and Special Postdoctoral Researcher of Hiroshima University.

Note added. -After submission of this manuscript, a related issue entitled by "observation of dynamical Casimir effect in a superconducting circuit" appeared. ${ }^{16}$

\section{References}

1. H. B. G. Casimir, Proc. K. Ned. Akad. Wet. 51, 793 (1948).

2. S. K. Lamoreaux, Phys. Rev. Lett. 78, 5 (1997).

3. U. Mohideen, et al., Phys. Rev. Lett. 81, 4549 (1998).

4. G. T. Moore, J. Math. Phys. 11, 2679 (1970).

5. A. Lambrecht, J. Opt. B: Quantum Semiclass. Opt. 7, S3 (2005).

6. M. Crocce, et al., Phys. Rev. A 70, 033811 (2004).

7. C. Braggio, et al., Europhys. Lett. 70, 754 (2005).

8. E. Segev, et al., Phys. Lett. A 370, 202 (2007).

9. V. V. Dodonov, V. I. Man'ko, and O. V. Man'ko, J. Sov. Laser Res. 10, 413 (1989).

10. K. Takashima, et al., J. Phys. A: Math. Theor. 41, 146036 (2008).

11. J. R. Johansson, et al., Phys. Rev. Lett. 103, 147003 (2009).

12. K. Takashima, et al., J. Phys : Conference Series 150, 052260 (2009).

13. T. Fujii, et al., Theoretical studies on dynamical Casimir effect in a superconducting artificial atom, in Proc. 9th Conf. Quantum Field Theory Under the Influence of External Conditions (QFEXT09), eds. K. A. Milton and M. Bordag (World Scientific, Singapore, 2010), p. 344

14. T. Fujii, et al., Phys. Rev. B 84, 174521 (2011).

15. M. Venkata Satyanarayana, Phys. Rev. D 32, 400 (1985).

16. M. Wilson, et al., Nature 479, 376 (2011). 